\title{
Perceptual relevance and art: some tentative suggestions.
}

\section{Patricia Kolaiti}

\begin{abstract}
A fundamental assumption in relevance theory is that human cognition has evolved in the direction of increased efficiency and, as such, tends, as Sperber and Wilson (1995: 38-46, 260-66) put it in their cognitive principle, to be naturally geared towards the maximisation of relevance. The cognitive principle inter alia explains the selectivity of human agency and attention: for an input to merit the attention of the human cognitive system, it must seem relevant enough to be worth attending to. But what makes an input relevant? The relevance-theoretic account, proposes that relevance for an individual organism at a time involves a balancing of mental effort and a particular type of positive or worthwhile modifications or effects, cognitive effects, that are representational in nature and amount to improvements in knowledge. The type of relevance yielded by such effects could be described as a cognitive type of relevance. However, inputs such as artistic stimuli -including literary ones- invite us to widen the scope of the causal engineering behind the selective directedness of our mental lives. Artistic stimuli merit the attention of the human cognitive system at various time-scales (momentary, developmental, evolutionary). Following Kolaiti (2019: 76-94) and drawing on neuroscientific evidence of the last 25 years, I will make tentative suggestions that artistic stimuli may also yield non-representational worthwhile modifications or effects. My discussion will focus on one such type of effects involving the human perceptual system: perceptual effects. Being partly or wholly embodied, perceptual effects could extend the machinery of relevance theory in an embodied direction and widen its interdisciplinary implications.
\end{abstract}

\section{Artistic stimuli as relevance-yielding occurrences}

A fundamental assumption in relevance theory (Sperber and Wilson 1995) is that human cognition has evolved in the direction of increasing efficiency. The human cognitive system tends, as Sperber and Wilson (1995: 38-46, 260-66) put it in their cognitive principle, to be naturally geared towards the maximisation of relevance, in managing its expenditures of mental effort and making the most productive use of its attentional and other resources. Relevance is technically defined as a relation between effort and effect such that the greater the (positive or worthwhile) effects of an input -assuming that effort remains constant- the greater its relevance for an individual at a time, and the smaller the mental effort required to process it -assuming that effects remain constant- the greater its relevance for an individual at a time.

The cognitive principle inter alia explains how human cognition avoids computational explosion. It explains why our cognitive systems do not pay the same amount of attention to each of the indefinite number of inputs out there in the world and/ or internal to our minds and bodies: that is, the indefinite number of facts that are perceptible or inferable at any time in our physical, bodily and mental surroundings. It also, and more crucially, explains why we attend to the particular facts that we do: for an 
input (be it a phenomenon or stimulus or area of human action and performance) ${ }^{1}$ to merit the attention of the human cognitive system, it must seem relevant enough to be worth attending to.

Sperber and Wilson would, therefore, say that in order to survive, public cultural stimuli have to hold inter-individual and collective attention and yield a good payoff for the processing effort required. Artistic phenomena and stimuli (including literary ones) merit the attention of the human cognitive system at various time-scales (momentary, developmental, evolutionary), to the point of art being among the most enduring and successful constituents of human public, cultural and mental life. Given the dual nature of art as an intra-individual and inter-individual occurrence, to ask why art merits the selectivity of human cognition is a twofold question: the one question is why public stimuli like artworks and literary texts merit the attention of human receivers; the other is why literary and artistic creation as a particularly laborious and effortful area of human action and performance merits the sustained attention and active engagement of human creators. From the viewpoint of Sperber and Wilson's cognitive principle, to say that an agent allocates steady and recurring attention on a process of literary and artistic creation is in other words to say that the agent derives considerable relevance from this process. But how is relevance to be understood in this case? And can the existing relevance-theoretic machinery account for all the ways artistic phenomena and stimuli are relevant to an individual organism at a given time?

In discussing what makes a phenomenon or stimulus relevant to an individual mind, Sperber and Wilson (1995) adopt the computational or representational Theory of Mind or ToM (Fodor 1975, 1981, 1983), according to which the facts that are manifest to an individual at a time and make up this individual's 'cognitive environment' or 'background' are computed and processed by the human mind in the form of mental representations, or propositions or sentences in the language of thought. ${ }^{2}$ In line with Fodor (1983), echoed in Sperber and Wilson (1995: 71-72), not all mental representations are conceptual representations. The representational theory of mind also covers linguistic (e.g. phonetic, syntactic, semantic), as well as perceptual (e.g. visual and auditory) representations. So, for relevance theory, the inputs to cognitive processes can be any type of representation -perceptual, sensorimotor, conceptual, etc. In this light Sperber and Wilson (1995: 153) note that:

A stimulus is a phenomenon designed to achieve cognitive effects. Relevance for a stimulus is thus the same as relevance for any other phenomenon (...).

\footnotetext{
${ }^{1}$ By 'phenomenon' I refer to any occurrence internal or external to the information-processing device, which occurs naturally or automatically or involuntarily and that the device processes as input. By 'stimulus' I refer to any occurrence external to the information-processing device designed to be perceived and that the device processes as input.

${ }^{2}$ Sentences in the language of thought are logical forms made up of concepts, and should not be thought of as synonymous with natural language sentences, which are made up of words.
} 
And relevance of a phenomenon is defined as follows:

\section{Relevance of a phenomenon (classificatory)}

A phenomenon is relevant to an individual if and only if one or more of the assumptions it makes manifest is relevant to him.

\section{Relevance of a phenomenon (comparative)}

Extent Condition 1: a phenomenon is relevant to an individual to the extent that the positive cognitive effects achieved when it is optimally processed are large

Extent Condition 2: a phenomenon is relevant to an individual to the extent that the effort required to process it optimally is small. (Sperber and Wilson 1995: 265-6).

It is clear from this quote that for Sperber and Wilson (1995) relevance involves a balancing of mental effort and a particular type of positive or worthwhile modifications or effects, cognitive effects. Sperber and Wilson (1995: 109) assume that within the range of possible modifications of the physical environment, there are cognitive modifications, and within the range of possible cognitive modifications, there are positive cognitive modifications which lead to improvements in knowledge: that is, to worthwhile cognitive effects. They then refine and illuminate their notion of cognitive effects by identifying the following three computationally traceable types of such effect:

a) More or less strongly or weakly evidenced contextual implications which result from interaction between new and old information used as premises in an inference process (Sperber and Wilson 1995: 109),

b) strengthening of old assumptions by new information which provides further evidence for them (Sperber and Wilson 1995: 109),

c) contradiction of old assumptions by new information which provides evidence against them and may lead to their abandonment or revision (Sperber and Wilson 1995: 109).

It is worth noting that Sperber and Wilson occasionally allude to the possibility that there may be more ways of achieving cognitive effects than the three mentioned above. In Relevance (1995: 66), for instance, there is a brief reference to other types of cognitive effect such as reorganisation of knowledge. Also, in 'Truthfulness and Relevance', Sperber and Wilson (2002: section 4) write:

Here we will consider only one type of cognitive benefit: improvements in knowledge (theoretical or practical). This is plausibly the most important type of cognitive benefit. There may be others: improvements in memory or imagination, for example (although it might be argued that these are 
benefits only because they contribute indirectly to improvements in knowledge; better memory and imagination lead to better non-demonstrative inference, and therefore to better knowledge).

In more recent work, Sperber and Wilson (2015) and Wilson (2018) use a broader notion of inference, encompassing a much wider range of inferential procedures compared to the one adopted in early work in relevance theory. On this broader view of inference (see Mercier and Sperber 2011 and 2017), cognition involves going well beyond the information available to the senses, integrating sensory stimulations at millions of nerve endings both to identify events in the environment and appropriately respond to these events by means of inference; memory and perceptual processes are therefore seen as involving a substantial element of inference, kinesic and sensorimotor mechanisms are treated as inferential, and relevance-yielding processes are explicitly referred to as inferential processes whose output is an indefinite array of propositions, with propositions construed in a much broader sense than that of natural language sentences so that they make room for the elicitation of images and phenomenal states. Sperber and Wilson (2015), Wilson (2018) and Wilson and Carston (2019) ${ }^{3}$ draw on this broader notion of inference in order to elaborate on ideas that were alluded to in early work in relevance theory (e.g. about indeterminacy of meaning, impressions consisting of a vast array of propositions etc.) and accommodate within a unitary account the three types of cognitive effects outlined earlier and a further type of so-called 'non-propositional' effects that often involve and are enabled by the parallel activation of perceptual/ sensorimotor and emotional mechanisms. In contrast with cognitive effects that are robustly propositional, 'non-propositional' effects do not constitute a meaning or message or import that can be rendered as a single proposition (or even small set of propositions) but amount to an indefinitely rich and complex array of propositions -with propositions construed in a much looser sense than that of natural language sentences, thereby making room for the evocation of unlexicalized ad hoc concepts, images and states of mind. ${ }^{4}$

This recent work by Sperber, Wilson and Carston accounts for one of the possible roles of perceptual/ sensorimotor and emotional mechanism activation during utterance interpretation, as well as for cases of embodied representationalism (Bolens 2012) in, say literary comprehension and interpretation. However, even this latter type of 'non-propositional' effects arising from the mobilisation

\footnotetext{
${ }^{3}$ It should be noted that there is nothing in this recent work by Sperber, Wilson and Carston that was not alluded to in the first edition of Relevance. So, their recent work is in fact an elaboration or development of earlier ideas, rather than something entirely new.

${ }^{4}$ It is important to distinguish here the claim that propositions evoke or are evoked by images -which relevance theory has always endorsed- from the claim that propositions consist of images, which relevance theory denies. In line with this, unlexicalised concepts can be part of propositions but images can't.
} 
of sensory, kinesic and affective information during utterance interpretation are still clearly a case of representational, conceptual and as such, propositional and hence, cognitive effects. ${ }^{5}$ The notion of 'nonpropositional' effects allows the explanatory mechanisms of relevance theory to account for embodied representationalism during utterance interpretation. The same could perhaps be argued for the neighbouring notion of poetic effects, developed within a relevance theory framework by Pilkington (2000). Still it could be argued that even these types of effects do not indicate a significant departure from the robustly cognitive approach of relevance theory, and Sperber, Wilson and Carston seem fully aware of that: the convention of using inverted commas to talk about 'non-propositional' effects captures the fact that the so-called 'non-propositional' effects are not truly non-propositional. Sperber, Wilson and Carston are in fact talking about a type of effect that perhaps would have been much better described as weakly propositional rather than 'non-propositional'. In this sense, the term 'non-propositional' effects is of little use in trying to account for possible types of genuinely non-propositional and non-cognitive worthwhile effects.

It could therefore be claimed that, as it stands, the relevance-theoretic approach implies an underlying view of human agents, ${ }^{6}$ human cognizers and human individual organisms as cognitive organisms. The current RT perspective, therefore, puts forth human individual organisms as cognitive organisms who selectively interact with inputs in the ecology of their natural, bodily, mental and cultural environment driven by expectations of cognitive effects and (cognitive) relevance guided principally by the cognitive system as a knowledge acquisition and knowledge management device. ${ }^{7}$ It is unclear from Sperber, Wilson and Cartson's existing writings, if this underlying view genuinely reflects their perspective of human individual organisms or if it is a contingent fact resulting from mere distribution of labour: to put it differently, it is unclear if relevance theory has so far discussed solely cognitive, propositional and representational types of effects and relevance because it believes that these are the only existing types of effects and relevance, or because it has merely divided labour by investigating the propositional and cognitive types of effects and relevance without nevertheless denying the possibility of non-propositional and non-cognitive ones.

In any case, from a literary- and art-philosophical point of view, a theoretical edifice that does not also involve non-cognitive types of effects and relevance could be seen as making the following two

\footnotetext{
${ }^{5}$ The same claim is made in Sperber and Wilson (1995: 24). And, in fact, this is precisely the reason why Sperber, Wilson and Carston have adopted the typographical convention of putting the term 'nonpropositional' effects in inverted commas.

${ }^{6}$ Where by 'human agent' I refer to an individual organism capable of deliberation and action.

${ }^{7}$ In this view, cultural artefacts such as artworks and literary texts are assumed to merit the selective directedness of our mental lives because they yield enough cognitive effects, i.e. enough improvements in knowledge for an individual organism at a time.
} 
predictions: a) that artworks and literary texts are stimuli of the exact same kind as mere utterances, i.e. ostensive stimuli (Sperber and Wilson 1995: 152-153), and b) that artworks and literary texts exhaust their raison d'etre and worth the attention of the individual organism principally or solely because they give rise to enough improvements in knowledge (cognitive effects) to offset the processing effort required. Both predictions can be shown to be counterintuitive. Let me explain.

The primary function of ostensive stimuli -e.g. utterances, gestures such as pointing or showing, imitations or demonstrations, etc.- is communicative: they are used to make an informative intention mutually manifest or in simpler words, let the audience know that the communicator intends to inform them of something. To achieve this goal, they must satisfy two conditions: they must attract the audience's attention and focus it on the communicator's informative intentions. Crucially for our discussion, Sperber and Wilson (1995: 152-153) propose that the best ostensive stimuli are entirely irrelevant unless they are treated as ostensive. Mere utterances are indeed ostensive stimuli. Mere utterances, like any ostensive stimulus, achieve relevance by attracting the audience's attention, focusing it on the communicator's intention and giving rise to improvements in knowledge by means of cognitive effects -and are indeed entirely irrelevant unless they are treated as ostensive. It should be quite uncontroversial that artistic stimuli fall at least partially within the scope of ostensive stimuli: artworks and literary texts may well be used as ostensive stimuli, i.e. used to make an informative intention mutually manifest and give rise to improvements in knowledge by means of cognitive effects. The problem with a theoretical account that does not predict other types of effects beyond cognitive ones is that, in such an account, artistic stimuli would be assumed to be relevant to the individual organism solely on the grounds of giving rise to improvements in knowledge by means of cognitive effects -since cognitive effects are the only type of effects that make a stimulus relevant to an individual organism. Now, given that this is precisely what ostensive stimuli do and given that artistic stimuli have an ostensive function, then artistic stimuli would be seen as fully accounted for by the notion of an ostensive stimulus: artistic stimuli would be seen as nothing more than an instance of ostensive stimuli. Given that the best ostensive stimuli are entirely irrelevant unless they are treated as ostensive, it would follow that artistic stimuli -being nothing more than ostensive stimuli- are entirely irrelevant unless they are treated as ostensive. This latter implication, however, appears counterintuitive: artistic stimuli seem to have intrinsic value of a certain kind. Unlike ostensive stimuli, artworks and literary texts appear to be perceptually and aesthetically pertinent to the organism independently of having or not having a parallel incidental ostensive function, they are pertinent to the 'senses', they are there to be experienced by and through the body, eliciting the fundamentally embodied type of characteristic experience we intuitively and pre-theoretically refer to as aesthetic experience. If so, then artistic stimuli cannot be adequately 
described as being nothing more than an ostensive stimulus and therefore, cannot be convincingly said to exhaust their raison d'etre simply and solely in giving rise to the types of effects (cognitive effects and improvements in knowledge -even in the sense of an indefinitely rich and complex array of weakly propositional types of effects-) that ostensive stimuli give rise to. My claim here is not that artworks and literary texts do not amongst other things give rise to improvements in knowledge or do not yield (cognitive) relevance and cognitive types of effects but that they are not principally, solely and necessarily relevant to human agents for any (cognitive) relevance and cognitive types of effects they might happen to yield. And if so, artworks and literary texts can be seen as exemplary case studies of alternative, non-representational reasons why various inputs might merit the sustained attention of human agents.

The picture becomes even more challenging if we shift the focus to literature and art as an action. ${ }^{8}$ It would be grossly counterintuitive to claim that the early laborious attempts of our ancestors tens of thousands of years ago to depict in the dimly lit recesses of their caves the animals that inhabited their world with a flair, artistry and astounding vivacity that went far beyond the merely functional and recreate details of shape, movement and form that would credit to artists of any epoch in the history of painting (Bahn 1998) were processes that merited the vast attentional effort of the creators mainly and solely because they gave rise to improvements in knowledge, i.e. to (cognitive) relevance and cognitive types of effects. To ask why literature and art merits the voluntary and involuntary attention of the human cognitive system as a type of human action and performance is in fact only a starting point in order to ask why many other domains of human action and performance merit the voluntary and involuntary attention of the human cognitive system.

I would now like to turn to literature and art in the light of recent neuroscientific and psychological findings to start making a number of tentative proposals about other possible types of effects and relevance beyond cognitive ones. I am currently in the process of applying for a three-year research project that will survey a wide array of pertinent neuroscientific findings from artistic creation and reception in order to attest these tentative assumptions and turn them into concrete suggestions. The aim of the present paper is not to offer definitive conclusions but to generate provisional, yet constructive, discussion in what I see as a new fascinating area of interdisciplinary investigation on the interface of literary and art study, relevance-theoretic pragmatics and the cognitive sciences.

\footnotetext{
8 Recent work in psychology and neuroscience (Allport 1987; Clark 2013, 2016, 2017; Hohwy 2012, 2013; Neumann 1987; Wu 2011a, b and 2014) emphasizes the crucial connections between attention, action and agency. The idea has long intellectual precedents going as far back as William James' (1890: 424) claim in The Principles of Psychology that 'volition is nothing but attention'.
} 


\section{Beyond cognitive relevance: artistic stimuli and perceptual effects}

Originating in the ancient Greek word 'aestheses', the notion of the aesthetic aims to capture the intuition that art and perception are somehow inexorably intertwined, that art even in its conceptually nuanced literary forms is primarily about the senses. Even a pre-theoretical understanding of an aesthetic experience as the characteristic kind of pleasurable perceptual and sensory response an agent has when experiencing an artwork is enough to shift our search for alternative types of positive or worthwhile effects and subsequent types of relevance that would be more appropriate to artistic stimuli in the direction of the human perceptual and sensorimotor system. Could we make a valid claim for perceptual types of worthwhile effects and how could they be determined?

In response to this question I would like to propose (in line with early suggestions made in my $\mathrm{PhD}$, Kolaiti: 2009) that a striking range of emerging findings in cognitive neuroscience and experimental psychology could be more adequately re-interpreted as describing different types of positive or worthwhile effects of artistic stimuli on the perceptual/ sensorimotor system. Let me briefly go through some pertinent findings.

In their article 'The Science of Art', Ramachandran and Hirstein (1999: 15-51), for instance, present a particular set of neuroscientific findings from the perceptual brain -with a particular focus on visual perception and the visual brain - and suggest that certain sets of visual stimuli seem to be more 'interesting' for the brain in the sense that the brain tends to allocate attention to them, preferring them over other competing sets of visual stimuli. The 'interesting' stimuli often elicit a pleasant 'aha!' sensation, caused perhaps by activation of the (limbic) reward system by the temporal lobe cortex. They then go on to propose that these interesting and inherently pleasing sets of visual stimuli can be grouped under eight heuristics or laws or principles, as they call them, that in their view are either consciously or subconsciously universally deployed by the visual arts to optimally activate the visual areas of the brain and can therefore be seen as a type of artistic universal. They call these universal heuristics 'Eight laws of artistic experience' and proceed to describing and grouping them as follows:

$-1^{\text {st }}$ law: The 'peak shift effect'

If a rat is taught to discriminate a rectangle from a square and is rewarded for the rectangle, the rat will tend to respond even more vigorously at the sight of a rectangle that is longer and skinnier than the prototype, i.e. a rectangle that has an exaggerated form of rectangularity. In Ramachandran and Hirstein's (1999: 15) view, the exaggerated rectangle functions like a super-stimulus and super-stimuli have been found to excite the visual brain more strongly than normal stimuli. This is the psychological phenomenon 
from which, as they suggest, not only caricatures, prehistoric fertility figures, African art and some of Picasso's work must have sprung but subsequently, much -if not all- of art.

$-2^{\text {nd }}$ law: Perceptual grouping and binding

According to Ramachandran and Blakeslee (1998) the process of discovering correlations and the process of grouping and binding the correlated features to create unitary objects or events must be reinforcing for the brain. Consider, for instance, the famous hidden Dalmatian dog task, initially seen as a jumble of splotches. The number of potential groupings of these splotches is infinite but once the splotches are grouped together in the correct sort of way in order to see the Dalmatian, a pleasant 'aha!' sensation is caused perhaps by activation of the limbic system by the temporal lobe cortex (Ramachandran and Hirstein 1999: 21). Perceiving a cubist painting, for instance, may be seen as involving similar universal grouping and binding operations. ${ }^{9}$

$-3^{\text {rd }}$ law: Isolating a single visual module and allocating attention

According to Ramachandran and Hirstein (1999: 24-25), isolating a single visual modality such as 'form' or 'depth' allows one to direct attention more effectively to this one modality and source of information. This explains why outlines are effective in art, in cases like line drawings, Indian art etc, while additional evidence for this view comes from the savant syndrome -autistic children who are "retarded" and yet produce beautiful drawings: '(...) this is because the fundamental disorder in autism is a distortion of the "salience landscape": they shut out many important sensory channels, thereby allowing them to deploy all their attentional resources on a single channel'.

$-4^{\text {th }}$ law: Discarding redundant information and extracting contrast

In line with Ramachandran and Hirstein (1999: 25), discarding redundant information and extracting contrast prior to grouping is also reinforcing: cells in the retina, the lateral geniculate body (a relay station in the brain that receives information from the retina) and the visual cortex tend to respond mainly to edges (step changes in luminance, regions of change) rather than to homogeneous surface colours, possibly because information exists mainly in such regions and it makes sense that they would, therefore, be more attention grabbing and interesting. Edges and contrast extractions are also intrinsically pleasing to the eye (hence the efficacy of cartoons and line drawings). A Monet or any other impressionist painting

${ }^{9}$ Ramachandran and Hirstein acknowledge that the idea that visual art deploys perceptual grouping and binding is not new but what they add in existing discussions on the matter is that the aesthetic experience that results from seen a visual artwork as pleasing must result from such physiological and neural operations. 
for that matter would be an excellent exemplar of discarding redundant information and extracting contrast.

$-5^{\text {th }}$ law: Symmetry

Unlike the previous laws, symmetry is not reinforcing in itself but attached to evolutionarily significant traits (Ramachandran and Hirstein 1999: 27): most biologically important objects such as predator, prey or mate are symmetrical and in this sense, the search for symmetry may be a perceptual mechanism geared towards discovering interesting biological objects in the world. At the same time, it has recently been shown experimentally that both animals and humans prefer symmetrical over asymmetrical mates and evolutionary biologists have argued that this is because parasitic infestation detrimental to fertility- often produces lopsided, asymmetrical growth and development. Symmetry is also aesthetically pleasing and Islamic art is a paradigmatic exemplar that Ramachandran and Hirstein draw on.

$-6^{\text {th }} \& 7^{\text {th }}$ law: The generic viewpoint and the Bayesian logic of perception

In line with Ramachandran and Hirstein (1999: 30), our visual system abhors interpretations which rely on a unique vantage point, it abhors suspicious coincidences. However a pleasing effect can be produced by violating this principle: 'For instance, there is a Picasso nude in which the improbability of the arm's outline exactly coinciding with that of the torso grabs the viewer's attention (...). (...) [Also] an object discovered after a struggle is more pleasing than one that is instantly obvious (...). (...) Perhaps the struggle itself is reinforcing (...).' (Ramachandran and Hirstein 1999: 30)

-8th law: Art as metaphor

Ramachandran and Hirstein's last law concerns art as metaphor. However, as I do not endorse the view of art as metaphor and also, adopt the deflationary relevance-theoretic account of metaphor as a case of loose use, I find Ramachandran and Hirstein's last law irrelevant to the present analysis and of a completely different order to their earlier suggestions. I will therefore not discuss it or consider it here.

The main thread, in my view, running through some of Ramachandran and Hirstein's findings is sub-attentive improvement of certain functions of the perceptual mind/ brain which are either evolutionarily significant in themselves (e.g. perceptual grouping and binding), or attached to other evolutionarily significant traits (e.g. symmetry). Ramachandran and Hirstein (1999) themselves refer to the modifications underlying their findings as 'reinforcing' for the perceptual brain. Even in those cases where conceptual/ cognitive outputs might also occur -the perceptual grouping and binding process, for 
instance, results in object recognition, i.e. in classifying the splotches as a Dalmatian, which is clearly a conceptual representation- Ramachandran and Hirstein's claim for perceptual brain reinforcement through attending to certain stimuli seems to me to still be a valid claim for at least two reasons: first, if we use the nature of the output as the sole determinant of the nature of the process then all processes should ultimately be thought of as enactivist. The ultimate output of perceptual, conceptual and affective processing is a more efficient engagement of human organisms with possibilities of action and a more sustainable and homeostatic exchange with their environment. Second, the key point in my view in Ramachandran and Hirstein's findings, just as in other findings from music and kinaesthetic perception that I will briefly refer to next, is that attending to certain stimuli improves and reinforces the ability of the perceptual brain to perform certain functions, independently of whether performing these functions might also trigger parallel conceptual activation and improvement of conceptual processes too. In fact, the composite nature of humans as organisms composed by complex interactions among a perceptual, conceptual and affective system makes rather self-explanatory the expectation that activation in the one system would elicit parallel activation in one, or both, of the other two systems (e.g. see Asari 2008 for evidence of heightened affective activation during unique perception tasks). ${ }^{10}$ But while it could be claimed that perceptual, conceptual and affective activation is always composite (that is, it is always in reality a case of co-activation), it could also be claimed that in different instances there is differential involvement and therefore differential potential improvement of each of the three systems. Ramachandran and Hirstein have not discussed why they describe their laws as perceptual brain reinforcing despite the fact that in some cases there is obvious involvement of conceptual cognition, but I think the answer is pretty straightforward: what they seem to suggest is that critical improvement as a result of attending to artistic stimuli occurs not so much in any perceptual or conceptual representations per se but in the perceptual brain's functions and processes through which these representations are yielded -from effectiveness in allocating attention to the speed with which a process is performed, etc.

Intriguingly, Ramachandran and Hirstein (1999: 25) suggest that processing artistic stimuli results in what we impressionistically experience as sensory pleasure, on the grounds that this exposure somehow contributes to or improves and reinforces the perceptual mind/ brain's aptitude for such processes. And that it may not be coincidental that what the brain cells find 'interesting' is also what the organism as a whole finds 'interesting', and perhaps in some circumstances 'interesting' for the brain translates into 'pleasing' for the organism as a whole.

\footnotetext{
${ }^{10}$ The interplay between perception and cognition is not a case of 'either or'; so, a relevant question here is whether there can be perceptual or other types of effects without a minimal at least activation of cognition. In Kolaiti (2009), I have made preliminary suggestions on the matter.
} 
Ramachandran and Hirstein can clearly be said, in my view, to have found a variety of worthwhile effects that are, nevertheless, of a perceptual rather than conceptual or at least, purely conceptual nature. I would therefore like to propose that Ramachandran and Hirstein's (1999) laws of aesthetic experience are better understood as describing different types of positive or worthwhile effects of the kind of stimulus literature and art is on the human perceptual/ sensorimotor system rather than as laws of some kind or other or as ontological claims about the essence of art, as Ramachandran and Hirstein seem to purport. Also, it seems that we are actually looking at many more types of perceptual effects than the ones identified in Ramachandran and Hirstein's laws. Recent neuroscientific research (Jola et al. 2011), for instance, has found that dancers show better integration of local proprioceptive signals than non-dancers, which can be attributed to perceptual effects resulting from engaging with the type of action and performance that dance is on the organism's ability to be in fine-grained informational states of a proprioceptive type. Similar evidence also emerges from the neuro-cognitive study of the 'expertise-effect' in music and dance (Calvo-Merino et al. 2005; Pantev et al. 2003; Putkinen et al. 2014; Tervaniemi 2001, 2009; Tervaniemi et al. 2015; Volpe et al. 2016) showing marked differential ability between experts and non-experts in sound and kinaesthetic perception tasks.

Although the allocation of attention on such phenomena and/ or stimuli and/ or types of action and performance might incidentally also give rise to parallel cognitive effects, the phenomena and/ or stimuli in question are nevertheless principally relevant to the organism because of the substantial worthwhile modifications that occur in the organism's perceptual/ sensorimotor organisation and functioning. They are therefore for the main perceptually relevant to the organism, yielding a type of relevance beyond the standard relevance-theoretic cognitive type: I will refer to it as perceptual relevance.

Perceptual effects, I would therefore suggest, constitute a plausible and neurologically real set of perceptual and sensorimotor system-improving modifications that can render an input relevant to an individual organism at a time, explaining -to some extent at least- why human agents find inputs like artistic stimuli and types of action and performance like art-creation rewarding enough to be worth attending to and revisiting or repeating. The existence of perceptual effects seems to capture in empirically and explanatorily tractable terms the intuition that the raison d'etre of the kind of stimulus an artwork is is to elicit a characteristic type of response or experience (aesthetic experience) that is fundamentally sensory or perceptual in nature. The fact, though, that perceptual effects do not occur exclusively in artistic phenomena and stimuli but also in non-artistic ones -e.g. in quiz games like the dog in splotches (see Ramachandran and Hirstein 1999)- which, although they elicit pleasurable 'aha' sensations, they nevertheless cannot be said to elicit full-blown aesthetic types of pleasurable experience, 
raises the question about whether artistic stimuli can be fully accounted for by perceptual relevance. Is it possible that in the case of art perceptual relevance has been further particularised into, say, an aesthetic variety of relevance whose machinery is perhaps very similar but not identical to that of generic perceptual relevance? I am only articulating this question at speculative level and do not purport to know the answer at this moment but certainly find this to be a fascinating area for future interdisciplinary research.

One other pertinent interdisciplinary question concerns whether there might exist more types of effects and relevance beyond cognitive and perceptual ones and how such effects might relate to artistic stimuli. In recent work, Wharton and Strey (2019) and Wharton and de Saussure (forthcoming) draw on Rey (1980), Cosmides and Tooby (2000) and Tooby and Cosmides' (2008) approach to emotions to address the relative ineffability of what they loosely call 'emotional communication'. By 'emotional communication' the authors refer to those vaguer aspects of communication discussed in Sperber and Wilson (2015) including descriptively ineffable emotional states that are too nebulous to be paraphrased without loss in finite propositional terms. Emotional communication is seen as operating on a number of different levels such as interjections, facial expression and affective tone of voice, which lead to either a single, determinate proposition or small set of propositions or contribute to altering the salience of a vast array of propositions (i.e. yielding a continuum of cases of cognitive effects of different strengths). Central to the authors argument about what is communicated by emotional communication is the idea that the traditional relevance-theoretic notion of positive cognitive effects needs to be complemented by a new notion of positive emotional effects. ${ }^{11}$ Although I have various complementary suggestions as far as their currently proposed definition of positive emotional effects is concerned, I nevertheless find the rationale that leads the authors to the suggestion that an affective type of effects might be theoretically necessary and psychologically and physiologically plausible both exciting and convincing. ${ }^{12}$ In fact I would tend to propose that if an affective type of effects is psychologically and physiologically plausible this would entail that an affective type of relevance of a phenomenon or stimulus for an individual organism at a given time should also be psychologically and physiologically plausible. I would also tend to propose that artistic stimuli could prove an exemplary case study in this front too, enabling a range of new insights that can be then transferred to other phenomena and stimuli such as emotional communication for instance. Emerging research (Bal and Veltkamp 2013; Djikik et al. 2013; Kidd and Castano 2013; Mar et al. 2006) on literary response and its temporary or long-term effects on empathy and the ToM capacity could be

\footnotetext{
${ }^{11}$ Similar suggestions having been made by Gutt (2013) and Strey (2016).

${ }^{12}$ Spelling out in detail my complementary suggestions about the possible content of an affective type of effects is not relevant to the aims of the present analysis.
} 
seen as a starting point for exploring possible types of affective effects of artistic stimuli, which can in turn provide empirical grounds for some aspects of extant affective theories of literature and art.

Finally, the investigation of perceptual effects could be extended by considering whether, perceptual effects identified in non-verbal artforms could perhaps find analogues in the conceptually nuanced medium of literature -i.e. natural human language: the idea here is that mobilisation of conceptual cognition (e.g. grouping and binding in conceptual terms) leads to activation of inner perception and mental imagery that could then yield 'literary analogues' of perceptual effects (e.g. grouping and binding in inner perception terms). Let me construct an example of a literary equivalent of a type of perceptual effect discussed by Ramachandran and Hirstein drawing on my own poetry work. In the poem Myasthenia Gravis I (Kolaiti 2007: 29) I write:

\author{
Now I must learn from scratch to spell tears \\ utter zeta and omega \\ and walk in spite of all \\ in the upright posture of humans.
}

The line 'utter zeta and omega' encrypts information that the reader can only uncover by 'binding correlated features to create a unitary object' in both perceptual and conceptual terms, to use Ramachandran and Hirstein's way of putting it. Zeta [Z] and omega [ $\Omega]$ are letters of the Greek alphabet which I have not, however, selected at random. When they are considered separately and independently of each other, the line allows a possible interpretation in which someone is learning again from scratch things as basic as the alphabet of her native language. But the two letters can also be notionally visualized and grouped together, in which case the reader will discover that they form a unitary object: the verb ' $Z \Omega$ '

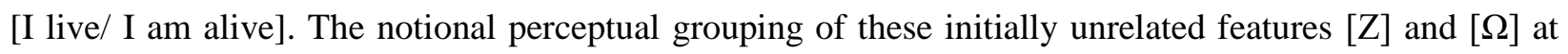
the level of inner perception allows a second potential, and deliberately 'hidden', interpretation involving learning from scratch what it means to be alive. It is worth stressing that the operations required by the reader in order to arrive at this second potential interpretation are both perceptual and conceptual and I therefore take this case as a clear literary equivalent of Ramachandran and Hirstein's laws of a) perceptual binding and grouping and b) object discovery following struggle in both perceptual and cognitive terms. Interestingly, the discovery that the two letters can also be mentally visualized and grouped together to form a unitary object elicits an introspectively evidenced pleasing 'aha!' sensation, similar to that found in perceptual tests like the 'hidden-face test' or 'the hidden-object test' mentioned in Ramachandran and 
Hirstein (1999: 21), although the final discovery here eventually concerns not perceptual but encyclopaedic features.

In similar terms, consider the following passage from Emily Dickinson discussed by Timothy Chesters (in Wilson 2018):

I stepped from plank to plank

So slow and cautiously;

The stars about my head I felt,

About my feet the sea.

'As Chesters' analysis shows, the chiasmus of 'Stars' ... 'felt' ...'feet'... 'sea' resulting from the syntax and prosody of the last two lines 'The stars about my head I felt / About my feet the sea' strives for balance at the very moment the speaker is most in danger of losing hers'.

What I find intriguing in this analysis, though, is that the chiasmus of 'Stars' ... 'felt'...'feet'...'sea' resulting from the syntax and prosody of the last two lines invite the reader to engage initially in a conceptual equivalent of extracting contrast prior to grouping -the minimalism of the poem encourages contrast extraction by foregrounding and facilitating the isolation of the corresponding concepts STARS, FEET and SEA allowing them to thereby behave as regions of change, as step changes in inner visual information in a way comparable to contrast extraction through step changes in luminance in an impressionist painting-, while prosody and syntax enable parallel binding and grouping operations that allow the reader by binding together STEPPED, STARS, FELT, FEET and SEA in the right sort of way to form in conceptual as much as inner-perceptual terms the unitary object of the heroin's body posture and disposition in space, both in terms of internal bodily disposition and awareness and external bodily disposition and awareness. ${ }^{13}$ The fact that the nature of the unitary object formed through these particular grouping and binding operations is a sensorimotor object, would lead me assume that the perceptual effects yielded by these two lines most possibly also involve mirror neuron function-based processes at the level of proprioceptive integration of kinaesthetic information, which in this case becomes available to the reader through inner kinaesthetic perception.

\footnotetext{
${ }^{13}$ What I mean by this is that the unitary perceptual object formed by binding together STEPPED, STARS, FELT, FEET and SEA elicits proprioceptive information both in terms of how the heroin feels and experiences the movement and posture and balancing of her body internally as well as in terms of what the heroin's movement and body posture and body balancing must look like if perceived from the outside, i.e. by an external observer.
} 
The investigation of the stunning wealth and diversity of ways in which literature across eras and cultures exploits mind-internal operations that yield perceptual effects ${ }^{14}$ may be seen as one of the paradigmatic fields of enquiry in which interdisciplinary interaction in the future could have precious two-way implications for research in both empirical and literary domains: the study of perceptual effects in literature, for instance, might illuminate one more causal mechanism behind the phenomenon of literary universals, i.e. the shared properties found across a range of geographically distinct literary traditions (Hogan 2003, 2011); at the same time, literature as much as art could be seen as a significant body of data for the way perceptual effects could be assumed to account for at least part of the causal engineering behind human selective directedness -i.e. the fascinating empirical question of how the mind selects what inputs to allocate attention to-, enabling new insights and hypotheses in affective and cognitive research about selectivity, attention and agency.

\section{Funding}

This project has received funding from the European Union's Horizon 2020 research and innovation programme under the Marie Skłodowska-Curie grant agreement 'CogLit' No [785964]

\section{References}

Allport, Alan 1987. Selection for action: Some behavioural and neurophysiological considerations of attention and action, in Perspectives on perception and action, A. Sanders and Heuer H. (eds.), Hillsdale, NJ: Lawrence Erlbaum Associates.

Asari, Tomoko, Konishi, Seiki, Jimura, Koji, Chikazoe, Junichi, Nakamura, Noriko and Miyashitaa, Yasusi 2008. Right temporopolar activation associated with unique perception. NeuroImage 41: 145-152.

Bal, Matthijs P. and Veltkamp, Martijn 2013. How Does Fiction Reading Influence Empathy? An Experimental Investigation on the Role of Emotional Transportation. PLOS ONE 8, 1: e55341. doi:10.1371/journal.pone.005534

Bolens, Guillemette 2012. The Style of Gestures: Embodiment and Cognition in Literary Narrative. Baltimore, MD: John Hopkins University Press.

Calvo-Merino, Beatrice, Glaser, Daniel E., Grèzes, Julie, Passingham, Richard E. and Haggard, Patrick 2005. Action observation and acquired motor skills: An fMRI study with expert dancers. Cerebral Cortex 15, 8: 1243-1249.

Clark, Andy 2013, Whatever next? Predictive brains, situated agents, and the future of cognitive science. Behavioral and Brain Sciences 36, 3:181-204.

Clark, Andy 2016. Surfing Uncertainty: Prediction, action, and the embodied mind. Oxford: Oxford University Press.

\footnotetext{
${ }^{14}$ I take my suggestions for future research to apply equally to affective types of effects.
} 
Clark, Andy 2017, Predictions, precision, and agentive attention. Consciousness and Cognition 56: 115119. doi: 10.1016/j.concog.2017.06.013

Cosmides, Leda and Tooby, John 2000. Evolutionary psychology and the emotions, in Lewis, M. and Haviland-Jones J. (eds.) Handbook of Emotions. New York: Guilford.

Djikic Maja, Oatley Keith and Moldoveanu Mihnea 2013. Reading other minds: Effects of literature on empathy. Scientific Study of Literature 3,1: 28-47. https://doi.org/10.1075/ssol.3.1.06dji

Fodor, Jerry 1975. The Language of Thought. Cambridge, MA: Harvard University Press.

Fodor, Jerry 1980. Methodological Solipsism Considered as a Research Strategy in Cognitive Science, Behavioral and Brain Sciences, 3: 63-73.

Fodor, Jerry 1981. Representations. Cambridge, MA: MIT Press.

Fodor, Jerry 1983. The Modularity of Mind. Cambridge, Massachusetts: MIT Press.

Fodor, Jerry 2000. The Mind Doesn't Work That Way. Cambridge, MA: MIT Press.

Gutt, Ernst August 2013. How does the affective relate to ostensive-inferential communication? Unpublished $m s$.

Hogan, Patrick Colm 2003. The Mind and Its Stories: Narrative Universals and Human Emotion. Cambridge University Press.

Hogan, Patrick Colm 2011a. What Literature Teaches Us about Emotion. Cambridge University Press.

Hohwy, Jacob 2012. Attention and Consciousness in the Hypothesis Testing Brain. Frontiers in Psychology 3: 96.

Hohwy, Jacob 2013. The Predictive Mind. Oxford: Oxford University Press.

James, William 1890. The Principles of Psychology. New York: Dover.

Jola, Corinne, Davis, Angharad and Haggard, Patrick 2011. Proprioceptive integration and body representation. Experimental Brain Research 213, 2-3: 257-265.

Jones Alice, Happé Francesca, Gilbert Francesca, Burnett Stephanie, Viding Essi 2010. Feeling, caring, knowing: different types of empathy deficit in boys with psychopathic tendencies and autism spectrum disorder. Journal of Child Psychology and Psychiatry 51,11: 1188-1197. doi: 10.1111/j.14697610.2010.02280.x

Kidd, David Comer and Castano, Emanuele 2013. Reading Literary Fiction Improves Theory of Mind. Science 342, 6156: 377-380, DOI: https://doi.org/10.1126/science.1239918

Kolaiti, Patricia 2007. Celesteia. Athens: Nefeli Publishings.

Kolaiti, Patricia 2009. The Limits of Expression: Language, Poetry, Thought. PhD Thesis, UCL. 
Kolaiti, Patricia 2019. The Limits of Expression: Language, Literature, Mind. Cambridge: Cambridge University Press.

Mar, Raymond A., Oatley, Keith, Hirsh, Jacob, de la Paz, Jennifer and Peterson, Jordan B. 2006. Bookworms versus nerds: Exposure to fiction versus non-fiction, divergent associations with social ability, and the simulation of fictional social worlds. Journal of Research in Personality 40, 5: 694-712. https://doi.org/10.1016/j.jrp.2005.08.002

Mercier, Hugo and Sperber, Dan 2011. Why do humans reason? Arguments for an argumentative theory. Behavioral and Brain Sciences 34: 57-111.

Mercier, Hugo and Sperber, Dan 2017. The enigma of reason. Cambridge, MA: Harvard University Press and Harmondsworth, UK: Penguin Books.

Neumann, Odmar 1987. Beyond Capacity: A functional view of attention, in Sanders A. and Heuer H. (eds.), Perspectives on perception and action Hillsdale, NJ: Lawrence Erlbaum Associates.

Pantev, Christo, Ross, Bernhard, Fujioka, Takako, Trainor, Laurel. J., Schulte, Michael and Schulz, Matthias 2003. Music and learning-induced cortical plasticity. Annals of the New York Academy of Sciences 999: 438-450.

Pilkington, Adrian 2000. Poetic Effects: A Relevance Theory Perspective. Amsterdam: John Benjamins.

Putkinen, Vesa, Tervaniemi, Mari, Saarikivi, Katri, Ojala, Pauliina and Huotilainen, Minna 2014. Enhanced development of auditory change detection in musically trained school-aged children: A longitudinal eventrelated potential study, Developmental Science 17, 2: 282-297.

Ramachandran, Vilayanur S. and Blakeslee, Sandra 1998. Phantoms in the Brain: Probing the Mysteries of the Human Mind. New York: William Morrow.

Ramachandran, Vilayanur S. and Hirstein, William 1999. The science of art: A neurological theory of aesthetic experience. Journal of Consciousness Studies 6: 15-51.

Rey, Georges 1980. Functionalism and the emotions, in Rorty, A. (ed.) Explaining Emotions. Los Angeles: University of California Press.

Sperber, Dan and Wilson, Deirdre 1995. Relevance: Communication and Cognition (2 ${ }^{\text {nd }}$ ed.). Oxford: Blackwell.

Sperber, Dan and Wilson, Deirdre 2015. Beyond speaker's meaning. Croatian Journal of Philosophy 15: 132.

Strey, Claudia 2016. The Language of Emotions. PhD thesis. Pontifical Catholic University of Rio Grande do Sul, Porto Alegre, BR.

Tervaniemi, Mari 2001. Musical sound processing in the human brain: Evidence from electric and magnetic recordings. Annals of the New York Academy of Sciences 930: 259-272. 
Tervaniemi, Mari 2009. Musicians: Same or different? Annals of the New York Academy of Sciences 1169: 151-156.

Tervaniemi, Mari, Janhunen, Lauri, Kruck, Stephanie, Putkinen, Vesa and Huotilainen, Minna 2015. Auditory profiles of classical, jazz, and rock musicians: Genre-specific-sensitivity to musical sound features. Frontiers in Psychology 6: 1900.

Tooby, John, and Cosmides, Leda (2008). The evolutionary psychology of the emotions and their relationship to internal regulatory variables, in Lewis, M., Haviland-Jones, J. M. and Barrett, L. F. (eds.) Handbook of emotions. The Guilford Press.

Volpe, Gualtiero, D’ Ausilio, Alessandro, Badino, Luciano, Camurri, Antonio and Fadiga, Luciano 2016. Measuring social interaction in music ensembles. Philosophical Transactions of the Royal Society B: Biological Sciences 371, 1693: 20150377.

Wharton, Tim and de Saussure, Louis (forthcoming) Pragmatics and Emotion. Cambridge: Cambridge University Press.

Wharton, Tim and Strey, Claudia (2019). Slave to the passions: Making emotions relevant, in Scott, K., Clark, B. and Carston, R. (eds.) Relevance: Pragmatics and Interpretation. Cambridge: Cambridge University Press. https://doi.org/10.1017/ 9781108290593

Wharton, Tim 2009. Pragmatics and Non-Verbal Communication. Cambridge: Cambridge University Press.

Wharton, Tim 2012. Pragmatics and prosody. In K. Allen and K. Jaszcolt, eds. The Cambridge Handbook of Pragmatics. Cambridge: Cambridge University Press.

Wharton, Tim 2016. That bloody so-and-so has retired. Lingua 175-176: 20-35.

Wilson, Deirdre and Carston, Robyn 2019. Pragmatics and the challenge of 'non-propositional' effects. Journal of Pragmatics 145: 31-38. https://doi.org/10.1016/j.pragma.2019.01.005

Wilson, Deirdre and Sperber, Dan 2002. Truthfulness and Relevance. Mind 111: 583-632.

Wilson, Deirdre and Sperber, Dan 2012. Meaning and Relevance. Cambridge: Cambridge University Press.

Wilson, Deirdre 2018. Relevance theory and literary interpretation, in Cave, T. and Wilson, D. (eds) Reading Beyond the Code. Oxford: Oxford University Press.

Wu, Wayne 2011a. Confronting many-many problems: Attention and agentive control. Noûs 45, 1: 51-60.

$\mathrm{Wu}$, Wayne 2011b. Attention as Selection for Action, in Mole, C., Smithies, D. and Wu, W. (eds) Attention: Philosophical and Psychological Essays. New York: Oxford University Press.

Wu, Wayne 2014. Attention. London: Routledge. 
